\title{
The Tau Trigger at the ATLAS Experiment
}

\author{
E. Ptacek ${ }^{* \dagger}$ \\ University of Oregon \\ E-mail: eptacek@uoregon.edu
}

The tau trigger system is a component of the ATLAS trigger system. Its purpose is to select events of interest containing hadronically decaying taus, which are signatures for new physics such as Higgs decays, while rejecting background events. It is composed of three levels, the lowest being hardware based and the higher levels software based. The tau trigger uses cuts on calorimeter and tracking information to select good tau candidates, the stringency of which cuts vary with luminosity. Tau trigger efficiency will be measured from the QCD tau fake rate, with a tag and probe analysis using $\mathrm{Z}$ boson decays, and from semi-leptonically decaying ttbar events triggered by a 4 -jet trigger.

XXth Hadron Collider Physics Symposium

November 16-20, 2009

Evian, France

\footnotetext{
${ }^{*}$ Speaker.

${ }^{\dagger}$ On behalf of the ATLAS TDAQ Collaboration.[1]
} 


\section{Introduction}

The purpose of the ATLAS trigger system is, in real time, to reduce the rate of events recorded from collisions in the detector, while keeping events of interest for physics analysis. It selects for offline storage event topologies which match signatures from a menu of triggers. Events (collisions) occur in the ATLAS detector at a rate of $40 \mathrm{MHz}$. The trigger system selects events at a rate compatible with the recording capacity of the ATLAS data acquisition system - approximately $200 \mathrm{~Hz}$. Each trigger of the system selects events with a specific signature. The signature for the ATLAS tau trigger is a tau that undergoes a hadronic decay. This signature is significant in a number of discovery physics channels. These include searches for the Minimum Supersymmetric Standard Model (MSSM) charged Higgs boson that undergoes the decay $H^{ \pm} \rightarrow \tau \nu$. These also include searches for final states with one or more tau leptons, such as the Standard Model Higgs boson, the MSSM neutral Higgs boson, and the Z' boson.[2]

\section{The ATLAS Trigger System}

The ATLAS trigger system[3] is composed of three levels. Level 1 (L1) of the trigger is hardware based. $\mathrm{L} 1$ reduces the event rate from $40 \mathrm{MHz}$ to $75 \mathrm{kHz}$. The predominant systems read by the the Level 1 trigger are the calorimeter and the muon spectrometer. Level 2 (L2) and the Event Filter (EF), which compose the High Level Trigger (the HLT), are software based. The HLT reduces the event rate from $75 \mathrm{kHz}$ to $200 \mathrm{~Hz}$. At the HLT data can, in principle, be accessed from the entire detector. HLT lepton and hadronic tau triggers access detector data from small regions, seeded by the particle candidates found in the L1 trigger. These Regions of Interest (RoI's) enlarge and increase in resolution from L1 to the EF. Events that pass the EF trigger

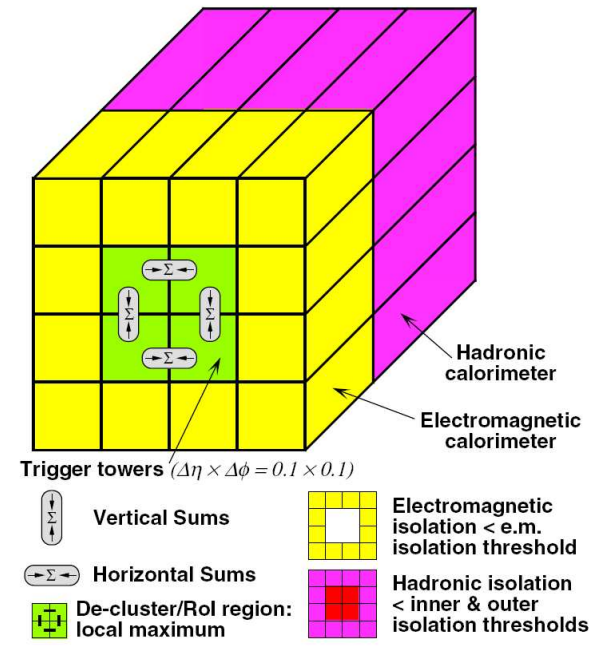

Figure 1: Diagram of trigger towers in the calorimeter. are written to offline storage.

\section{The Tau Trigger System}

The tau trigger system uses calorimeter and tracking information to accept signals from tau jets (hadronically decaying taus) while rejecting background from QCD jets. The tau jet from a hadronic tau decay is primarily one or three charged pions, a neutrino and possibly neutral pions. At Level 1 the tau trigger signature is a localized peak in energy in the electromagnetic and hadronic calorimeter. This peak is isolated using a 4 by 4 sliding window of trigger towers with a granularity of 0.1 in eta and phi.[4] Beginning at L2, the HLT reprocesses, with increased resolution, the regions of the calorimeter surrounding the Level 1 seeds, the L1 RoI's. It generates a more refined measurement of the cluster energy by using calorimeter cells, which have a finer granularity than the trigger towers used at L1. Cuts are made based on shower shape variables. 
The HLT also utilizes tracking information as a criteria for trigger acceptance. The three most significant tracking-based cuts are: the minimum transverse momentum $(\mathrm{Pt})$ of the leading track; the maximum energy deposited in an isolation ring around the tau candidate relative to the energy deposited in the central core area; and the maximum number of low Pt tracks in the RoI. [2] By the final stage of the HLT, the Event Filter, the accuracy of the tau reconstruction is close to that of the full offline reconstruction, as the EF algorithms are based closely on the offline tau reconstruction algorithms. The primary source of fake tau triggers are QCD jets. The tau trigger uses the characteristic low track multiplicity and narrowness of the tau jet and the isolation of the tau jet energy peak in the calorimeter to reject QCD jets.

\section{The Tau Trigger Menu}

The tau trigger menu, which depends on luminosity, is composed of both single tau and combined tau triggers. The tightness of the selection criteria used in selecting tau signatures will depend on luminosity. Tighter selection criteria will be used for higher luminosities and looser for early runs at lower luminosities. Due to the high fake rates in single tau triggers, taus of moderate $\mathrm{Pt}$ will primarily be selected by combined triggers. These include the tau + lepton (+jets), the tau + MET (Missing Transverse Energy), and the tau + tau trig-

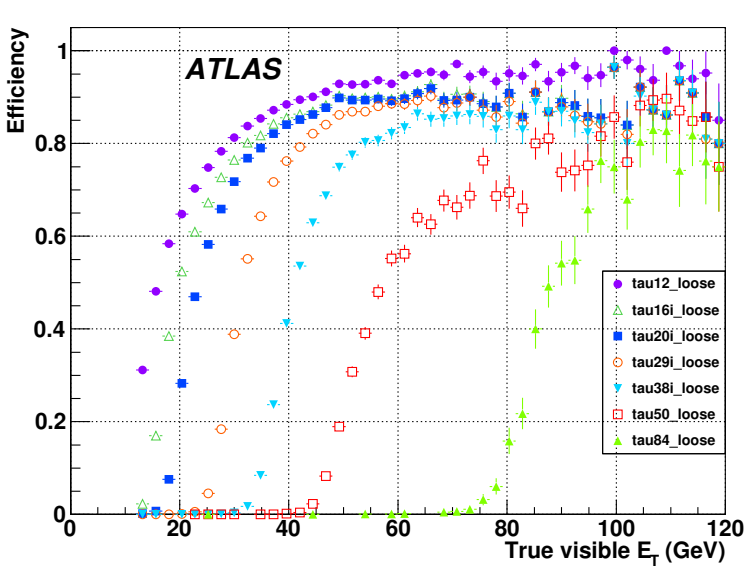

Figure 2: For various triggers, trigger efficiency on truth matched offline reconstructed tau candidates from MC simulation of $Z \rightarrow \tau \tau$ decay. gers. Table 1 shows a selection of tau signatures for early running with a luminosity of $10^{31} \mathrm{~cm}^{-2} \mathrm{~s}^{-1}$. The name of the trigger reflects the Pt threshold at EF and the strictness of the cuts used by the trigger in passing the events; "loose" refers to the weakest cuts. The symbol "xe" refers to a trigger based on missing transverse energy (MET). The trigger rates are calculated using Monte Carlo simulation.[5]

\section{Tau Trigger Efficiency Measurement}

The tau trigger efficiency will be measured with respect to the offline tau reconstruction in early data. Trigger efficiency with respect to offline reconstruction is the probability that the trigger

Table 1: Tau trigger menu for $L=10^{31} \mathrm{~cm}^{-2} \mathrm{~s}^{-1}$; for running at $10 \mathrm{TeV}$

\begin{tabular}{|l|l|l|l|} 
Menu & Goal & Trigger & Unprescaled Rate $[\mathrm{Hz}]$ \\
\hline Single Tau & Searches at high $P_{T}$ & tau50loose & 0.9 \\
tau+lepton & $Z \rightarrow \tau \tau$ & tau12loose + e10loose & 2.2 \\
tau+MET & $W \rightarrow \tau \nu, t \bar{t}, Z \rightarrow \tau \tau$ & tau16iloose + xe30 & 2.4 \\
tau+(b)jets & $t \bar{t}$ & tau16iloose + 3j40 & 1.1
\end{tabular}


accepts an event given that a tau candidate was reconstructed by the offline algorithms. There are a number of methods for the measurement of trigger efficiency. A simple estimate of tau trigger efficiency can be made by counting tau triggers on all taus that are reconstructed offline (composed mostly of fake taus, mimicked by QCD jets). In the medium Pt range (30 to $60 \mathrm{GeV}$ ) a more refined measurement will be made using a tag-and-probe method which selects $\mathrm{Z}$ boson events. The $\mathrm{Z}$ boson decays to taus and leptons (electrons and muons) and neutrinos. Events are selected with single object electron or muon triggers (tag side). The tau trigger efficiency is then determined on the other side (probe side) for tau leptons reconstructed and identified offline. Top events will be used to measure efficiency for the tau + MET trigger. In semi-leptonically decaying ttbar events, triggered by a 4-jet trigger, the tau and neutrino can be used as a probe for the trigger efficiency measurement.[6] The efficiency for high Pt tau triggers will be measured indirectly, using a bootstrap method in which a measurement of the relative efficiency of low vs high Pt triggers is used to calculate the efficiency of the high Pt trigger from the measured efficiency of the low $\mathrm{Pt}$ trigger.

\section{References}

[1] The ATLAS Collaboration, The ATLAS Trigger DAQ Authorlist, ATL-DAQ-PUB-2009-008, Geneva, 2009

[2] The ATLAS Collaboration, Tau Trigger: Performance and Menus for Early Running, ATŁ-PHYS-PUB-2009-000

[3] The ATLAS Collaboration, The ATLAS Experiment at the CERN Large Hadron Collider, 2008 JINST 3 S08003

[4] ATLAS L1Calo Group, compiled by E. Eisenhandler, ATLAS Level-1 Calorimeter Trigger Algorithms, ATL-DAQ-2004-011

[5] Andreas Reinsch, The Tau Trigger at the ATLAS Experiment, ATL-DAQ-PROC-2009-027

[6] A. Reinsch, The ATLAS TDAQ Collaboration, Tau Trigger at the ATLAS Experiment, ATL-DAQ-SLIDE-2009-221 